\title{
Anne A. LATOWSKY, Emperor of the World: Charlemagne and the Construction of Imperial Authority, 800-1229
}

\section{Sverre Bagge}

\section{OpenEdition}

\section{Journals}

Édition électronique

URL : https://journals.openedition.org/ccm/5179

DOI : $10.4000 / \mathrm{ccm} .5179$

ISSN : 2119-1026

\section{Éditeur}

Centre d'études supérieures de civilisation médiévale/Université de Poitiers

\section{Édition imprimée}

Date de publication : 1 septembre 2020

Pagination : 205-206

ISBN : 978-2-490783-06-9

ISSN : 0007-9731

Référence électronique

Sverre Bagge, «Anne A. LATowsky, Emperor of the World: Charlemagne and the Construction of Imperial Authority, 800-1229 », Cahiers de civilisation médiévale [En ligne], 250-251 | 2020, mis en ligne le 01 septembre 2021, consulté le 24 novembre 2022. URL : http://journals.openedition.org/ccm/5179 ; DOI : https://doi.org/10.4000/ccm.5179

\section{(c) (i) (3)}

Creative Commons - Attribution - Pas d'Utilisation Commerciale - Pas de Modification 4.0 International - CC BY-NC-ND 4.0

https://creativecommons.org/licenses/by-nc-nd/4.0/ 
Anne A. Latowsky, Emperor of the World: Charlemagne and the Construction of Imperial Authority, 800-1229, Ithaca/Londres, Cornell University Press, 2013.

Le livre d'Anne A. Latowsky traite de l'Histoire imaginée, l'histoire de Charlemagne visitant Jérusalem et Constantinople, étant reconnu comme l'empereur du monde. Il s'inscrit donc dans une tendance marquée de la littérature contemporaine en retraçant les origines et le développement des mythes et des idéologies qui ont influencé la culture et la société médiévales. Le point de départ de A. A. Latowsky est le chap. 16 de la Vita Caroli Magni d'Einhard sur Charles en tant que protecteur des lieux saints, et qui reçoit des ambassades et des cadeaux de dirigeants de pays lointains tels que Byzance et le monde arabe, dont l'exemple le plus spectaculaire est l'éléphant offert en cadeau par Harun al-Rashid. Elle discute du contexte général du passage et de son lien avec le renouveau classique de la période carolingienne, avant de traiter sa transformation progressive en une idée d'empire mondial fondé par Charlemagne lors de sa visite en Orient, et de l'importance de cette idée pour l'idéologie impériale.

A. A. Latowsky commence par montrer comment le bref chapitre d'Einhard est développé dans des écrits plus tardifs sur Charlemagne jusqu'à la fin $d u X^{e} s$. Puis il y a une éclipse d'une centaine d'années, jusqu'à la compilation d'un texte sur la façon dont Charlemagne a reçu des reliques de la Passion, en provenance de Constantinople, et les a ramenées en France. Ce texte, généralement appelé Descriptio, marque une nouvelle époque et appartient à un contexte complètement différent de celui que les érudits avaient maintenu jusqu'alors. Dans sa forme actuelle, il provient de France et célèbre 
les reliques appartenant au monastère Saint-Denis. Daté de v. 1080, il semblerait être lié à l'idée de croisade, ce qui était aussi la première hypothèse de A. A. Latowsky. Cependant, celle-ci précise que la plus grande partie du texte dérive d'un texte antérieur appartenant à un contexte différent : celui de l'idée d'un empire universel. Le voyage de Charlemagne vers l'est se fait paisiblement. Il refuse même d'accepter les cadeaux de l'empereur byzantin, avant de finir par les accepter. Le pape ne joue aucun rôle dans cet empire, contrairement à ce qui est dit dans les versions précédentes, où il donne la couronne impériale à Charlemagne et agit ainsi comme son protecteur. Ainsi, le Descriptio forme un nouveau départ : les versions suivantes appartiennent au contexte de l'idéologie impériale allemande, telle que développée depuis la querelle des Investitures et après, notamment sous Frédéric Barberousse.

A. A. Latowsky consacre un chapitre entier au propagandiste le plus important de cette idéologie, pendant cette période de querelle des Investitures : Benzon d'Albe. Il a composé une série d'œuvres en vers et en prose, dans lesquelles il célèbre le pouvoir impérial sous Henri III et Henri IV. Il utilise la légende de Charlemagne pour défendre l'empire contre le pape et les Normands : Charlemagne n'est plus le protecteur du pape, il est le souverain qui a écrasé les Saxons (aussi ennemis d'Henri IV), vaincu les Lombards en Italie et reçu des dons symboliques d'Orient pour rétablir l'Empire romain. La tradition de Benzon se poursuit et se développe sous Frédéric Barberousse, dont le règne est couvert par deux chapitres du livre qui traitent une série d'auteurs et de textes issus de l'entourage de l'empereur : Otto de Freising, Rahewin, le Ludus de Antichristo, la chancellerie impériale sous Renaud de Dassel, l'Archipoète et Godefroi de Viterbe. Alors que Charlemagne a joué un rôle subalterne pendant les premières années du règne de Frédéric, comme expliqué en particulier dans la Gesta Frederici d'Otto de Freising, il n'est devenu une figure centrale qu'à partir de 1160 environ, après le schisme papal et le soutien de Frédéric à l'anti-pape Victor IV, un développement qui a atteint son apogée avec sa canonisation en 1165. Ayant ainsi présenté le développement de la légende de Charlemagne en Orient, A. A. Latowsky se tourne au chap. 6 vers ce qui était son projet originel : Charlemagne en France pendant la période des Croisades, montrant l'empereur comme un modèle pour les rois croisés français, et notamment Saint Louis. Dans le dernier chapitre, elle retourne une fois de plus en Allemagne et ajoute un épilogue sur Frédéric II, couronné à Aix-la-Chapelle, qui a dirigé la translation des restes de Charlemagne et achevé la capitulation pacifique de Jérusalem en 1229.

A. A. Latowsky relie ainsi la légende de Charlemagne en Orient aux enjeux politiques cruciaux des $\mathrm{XI}^{\mathrm{e}}$ et $\mathrm{XII}^{\mathrm{e}} \mathrm{s}$. Ses arguments en ce sens ne sont pas toujours faciles à suivre, en particulier le passage sur le Descriptio qui rend la lecture difficile. Dans son ensemble, cependant, elle présente une analyse intéressante et vraiment convaincante. En me basant sur ma propre lecture d'Otto de Freising, je peux toutefois suggérer qu'elle a peut-être exagéré le contraste entre Otto et les écrivains postérieurs au service de Frédéric Barberousse. Dans sa Chronica, Otto énumère une série continue d'empereurs romains, de l'Antiquité classique jusqu'à son époque, à partir du règne de Constantin et ses successeurs à Constantinople, mais du règne de Charlemagne à l'ouest. Il est tentant de combiner cela avec le célèbre discours de Frédéric aux romains dans le Livre II de la Geste, lorsqu'il prétend que la République romaine, que les citoyens de Rome croient avoir restaurée, s'est effectivement déplacée vers « les Francs », c'est-à-dire le peuple gouverné par Frédéric. Otto ne dit pas quand cela s'est produit mais, sur la base de la Chronica, il semblerait probable qu'il pensait à Charlemagne. En outre, Otto est également extrêmement préoccupé par la relation avec Byzance, citant même, et contrairement à son habitude, une longue correspondance entre Conrad III et Manuel I ${ }^{\text {er }}$ Comnène sur le droit au titre d'Empereur. Ainsi, indirectement, Otto a peut-être attribué une grande importance à Charlemagne, bien que sans partager l'idéologie anti-papale de Benzon d'Albe et des écrivains impériaux pendant le schisme papal.

Certaines des conclusions de A. A. Latowsky peuvent donc être sujettes à discussion, sans que cela ne nuise à la valeur de son livre. De manière générale, le livre de A. A. Latowsky est passionnant et convaincant. Il donne un sens à nombre de textes fantastiques et obscurs en les reliant aux questions politiques majeures de cette période. Son analyse est un bon exemple de la façon dont les sources qui contiennent des faits à peine fiables peuvent jeter un nouvel éclairage sur la pensée politique médiévale et l'interprétation de l'Histoire.

Sverre BAGGE Université de Bergen 